\title{
Clinical and genetic spectrum of an orphan disease MPAN: a series with new variants and a novel phenotype
}

\author{
Nihan Hande Akçakaya ${ }^{1,2}$, Garen Haryanyan², Sevcan Mercan², Nejla Sözer ${ }^{3}$, Asuman Ali ${ }^{4}$, \\ Temel Tombul ${ }^{5}$, Uğur Özbek ${ }^{6}$, Sibel Aylin Uğur İşeri ${ }^{2}$, Zuhal Yapıc1 ${ }^{7}$ \\ ${ }^{1}$ Council of Forensic Medicine, Kımız sokak no:1 Bahçelievler, Istanbul, Turkey \\ ${ }^{2}$ Department of Genetics, Institute of Aziz Sancar Experimental Medicine (ASDETAE), Istanbul, University, Istanbul, Turkey \\ ${ }^{3}$ Department of Neurology, Dr. Sadi Konuk Training and Research Hospital, Health Sciences University, Istanbul, Turkey \\ ${ }^{4}$ Department of Neurology, Yuksek Ihtisas Training and Research Hospital, Health Sciences University, Bursa, Turkey \\ ${ }^{5}$ Department of Neurology, Yuzuncu Yil Faculty of Medicine, Yuzuncu Yil University, Van, Turkey \\ ${ }^{6}$ Department of Medical Genetics, Acibadem Faculty of Medicine, Acibadem University, Istanbul, Turkey \\ ${ }^{7}$ Department of Child Neurology, Istanbul University, Istanbul, Turkey
}

\begin{abstract}
:
Introduction. Pathogenic variations in C19orf12 are responsible for two allelic diseases: mitochondrial membrane protein-associated neurodegeneration (MPAN); and spastic paraplegia type 43 (SPG43). MPAN is an orphan disease, which presents with spasticity, dystonia, peripheral nerve involvement, and dementia. The pattern of iron accumulation on brain MRI may be a clue for the diagnosis of MPAN. SPG43, on the other hand, is characterised by progressive lower limb spasticity without brain iron accumulation. We here present clinical and genetic findings of MPAN patients with potentially pathogenic C19orf12 variants.

Materials and methods. Patients from 13 different families having progressive motor symptoms with irritative pyramidal signs and brain iron accumulation were screened for C19orf12 gene variants.

Results. C19orf12 screening identified seven variants associated with MPAN in eight patients from seven families. We associated two pathogenic variants (c.24G > C; p.(Lys8Asn) and c.194G > A; p.(Gly65Glu)) with the MPAN phenotype for the first time. We also provided a genetic diagnosis for a patient with an atypical MPAN presentation. The variant c.32C $>T ; p$.(Thr11Met), common to Turkish adult-onset MPAN patients, was also detected in two unrelated late-onset MPAN patients.

Conclusions. Genetic analysis along with thorough clinical analysis supported by radiological findings will aid the differential diagnosis of MPAN within the neurodegeneration with brain iron accumulation spectrum as well as other disorders including hereditary spastic paraplegia. Dystonia and parkinsonism may not be the leading clinical findings in MPAN patients, as these are absent in the atypical case. Finally, we emphasise that the existence of frameshifting variants may bias the age of onset toward childhood.
\end{abstract}

Key words: MPAN, C19orf12, SPG43, iron accumulation, spastic paraplegia, HSP

(Neurol Neurochir Pol 2019; 53 (6): 476-483)

\section{Introduction}

Neurodegeneration with brain iron accumulation (NBIA) comprises a group of rare inherited movement disorders accompanied by varying degrees of cognitive dysfunction. The combination of extrapyramidal motor system involvement and iron accumulation at the basal ganglia on brain magnetic resonance imaging (MRI) leads simply to a suspicion of an NBIA disorder. However, identifying the distinct NBIA subtype can be challenging due to clinical and genetic heterogeneity $[1,2]$. 
Mitochondrial membrane protein-associated neurodegeneration (MPAN) is the third most frequent subtype of NBIA (MIM:614298). MPAN, also known as NBIA4 and first defined in a Polish NBIA cohort, comprises $38 \%$ of total cases [1]. The C19orf12 protein shows mitochondrial localisation. Lewy bodies, tangles, spheroids, and tau pathology were present in histopathological examination of the brain in an autopsy case [1]. The major clinical features of MPAN are spasticity, dystonia, peripheral nerve involvement, and dementia [1]. The prominent clinical features of MPAN may show a great variability among patients, such as manifesting severe peripheral motor axonal neuropathy or amyotrophic lateral sclerosis mimicking clinical progress [1-3].

The age of MPAN onset can vary from childhood to adulthood. The earliest onset of MPAN has been reported at the age of 3 , and the oldest at the age of $36[3,4]$. Initial symptoms involve movement abnormalities, behavioural disturbances, cognitive impairment, optic atrophy, motor axonal neuropathy, and bladder incontinence [5]. Gait problems and spasticity, predominantly in the lower limbs, are also early symptoms of MPAN [1]. In early onset MPAN cases, dystonia and spasticity comprise the most common motor symptoms. Parkinsonism is mostly observed in cases with a later onset. Emotional lability, anxiety, compulsions, depression, impulsiveness and psychosis are the non-motor symptoms [5]. The rate of progression of MPAN is not clearly defined, but adulthood onset cases can show an aggressive progression leading to death [4-6].

The radiological spectrum of MPAN is also heterogeneous, without a distinct MRI sign. Nevertheless, the main MRI finding is iron accumulation at globus pallidus (GP) equally with substantia nigra (SN) $[1,5]$. However, iron accumulation may be undetectable in the early stages of MPAN [7]. Medial medullary lamina linear streaking on T2 sequences is an important sign supporting a differential diagnosis of MPAN [5]. Cerebral and cerebellar atrophy may be observed in the late stages [5]. Rare cases have been reported with a so-called 'eye-of-the-tiger' pattern in MRI, defined as hyperintensity in the anteromedial area of GP surrounded by low-signal intensity rings on a T2-weighted MRI $[7,8]$. This MRI pattern is typical for pantothenate kinase-associated neurodegeneration (PKAN), which is the most common subtype of NBIA $[1,9]$.

The pathogenic variations in C19orf12 lead to MPAN through a possible defect affecting fatty acid biosynthesis and branched-chain amino acid metabolism $[1,10]$. C19orf12 is also responsible for another allelic disease, namely hereditary spastic parapalegia (HSP) type 43 (MIM: 615043). HSP is basically an umbrella term for different rare neurodegenerative diseases that feature progressive spasticity of the lower limbs. Brain iron accumulation is not obvious in HSPs. Below, we present genetic findings of eight patients from seven Turkish families, along with their clinical and neuroimaging findings. We discuss the implications on the MPAN phenotype.

\section{Patients and methods}

Fourteen patients from 13 different families having progressive motor symptoms with irritative pyramidal signs and iron accumulation in basal ganglias were recruited in this study for a possible genetic diagnosis of MPAN (Supplementary Tab.1). All patients had previously tested negative for pathogenic variations in PANK2 associated with PKAN. Informed consent was obtained from the patients and family members in accordance with the ethics approval obtained for the study from the Ethics Committee of Istanbul University, Istanbul Faculty of Medicine.

\section{Genetic analysis}

Genomic DNA was isolated from peripheral blood using standard protocols. All exons and exon-intron boundaries of the C19orf12 transcript variant NM_001031726.3 (ENST00000392278.2) coding the largest protein isoform were investigated for sequence variations using PCR and Sanger sequencing protocols. This reference transcript was also used to perform all variant annotations. Segregation analysis was performed in available family members upon identification of a potentially causative variation. Primer sequences are given in the supplementary data. PCR conditions are available upon request. Sequence data was analysed using a CLC Main Workbench v6.5. (CLC Bio, Aarhus, Denmark). The frequency of identified variations was evaluated through the Genome Aggregation Database (GnomAD). All variants were annotated with an ENSEMBL-Variant Effect Predictor to identify all possible consequences on RNA and protein structure and also to evaluate with in silico tools, namely SIFT and Polyphen.

\section{Results}

We detected seven different potentially pathogenic C19orf12 variants in eight patients from seven families. A review of the clinical and genetic findings of these patients is set out in Table 1 . Two of these variants were associated with MPAN for the first time in this study, including c.194G > A in rs752450983 locus and one novel variant c. $24 \mathrm{G}>\mathrm{C}$. These two variants were submitted to the freely accessible NCBI ClinVar Database, with SUB4966204 and SUB4967569 submission numbers respectively. All variants were in the homozygous state except for the variants of two siblings in Family 3, which were confirmed to be in a compound heterozygous state by segregation analysis. c.32C > T; p.(Thr11Met) (rs397514477), which has previously been reported to be common among adult Turkish patients with MPAN, was found to be homozygous in two unrelated patients [4]. Figure 1 shows three selected variants for which segregation analysis was possible.

Interestingly, another patient (M8) was found to be heterozygous for a single variant in C19orf12 gene, which 


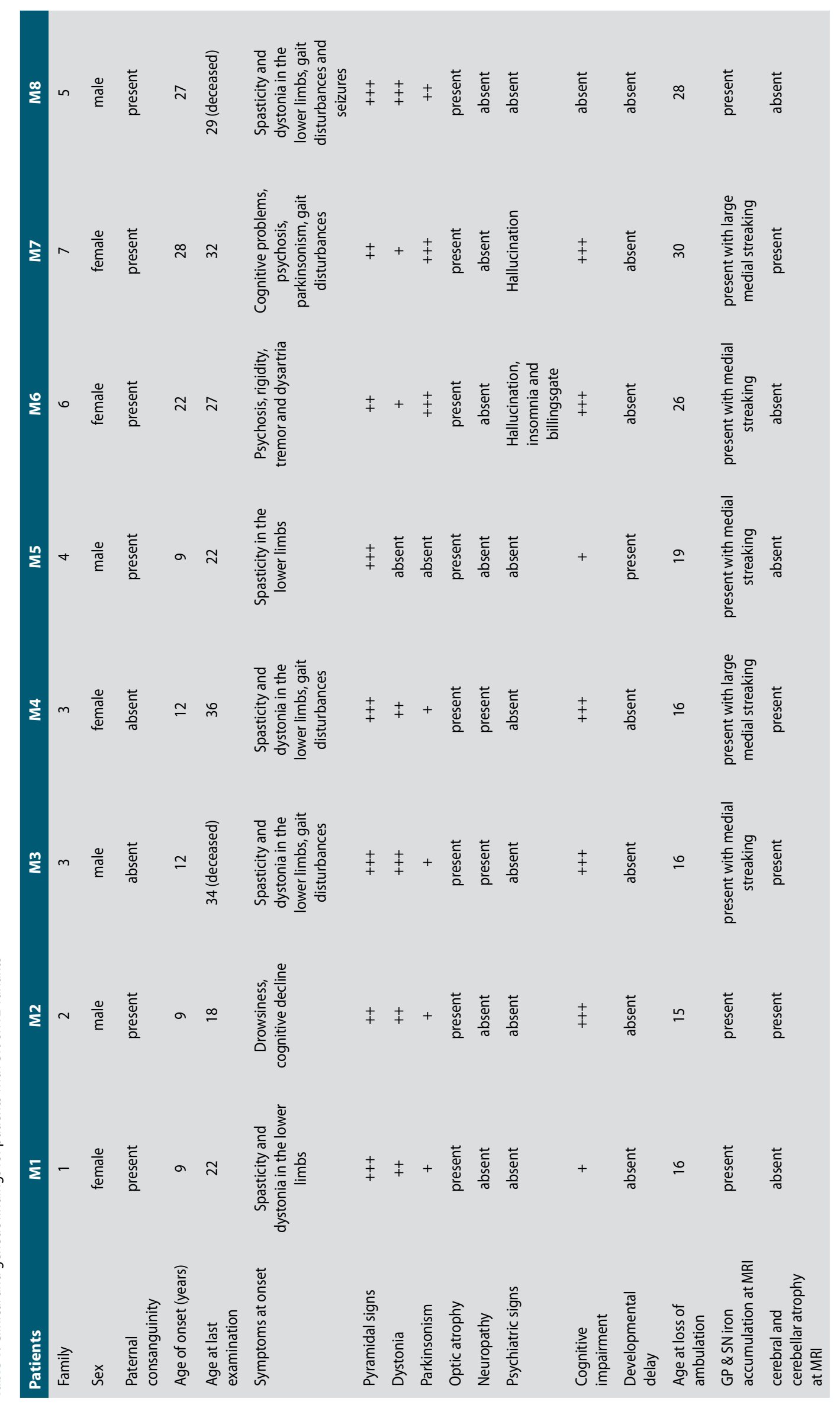




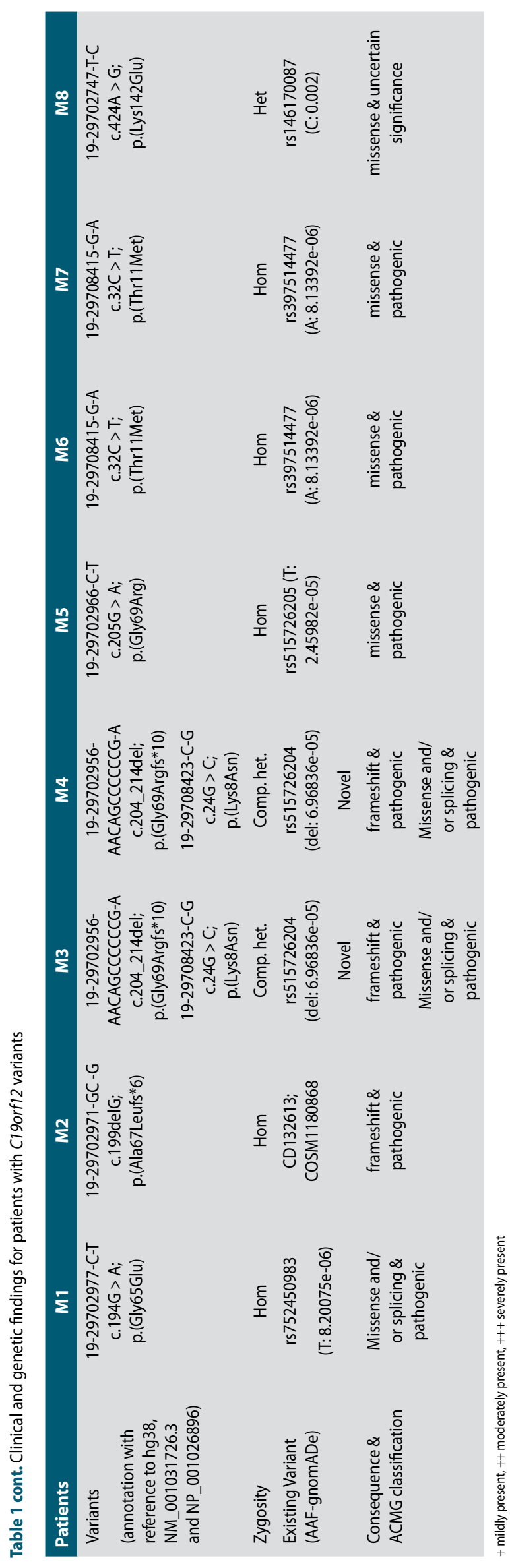

has conflicting predictions of pathogenicity in ClinVar (rs146170087). Although the alternative allele frequency $(\mathrm{AAF}=0.002)$ is relatively high compared to other variants identified in this study, it still fits the carrier frequency estimation of MPAN (carrier frequency range of $0.002-0.005$ for MPAN with a prevalence of 1-9:1,000,000 from Orphanet; ORPHA:289560). However, the variant was in a heterozygous state and another accompanying allele was not detected in any C19orf12 regions analysed.

The age of onset for patients with MPAN variants ranged from 9 to 27 years (Tab. 1). The most frequent clinical presentation was lower extremity-onset spasticity with dystonia (5/8). All patients had dystonia except for M5. The phenotype of patient M5 was submitted to the NCBI ClinVar Database with the SUB4967579 submission number. Among adult-onset MPAN patients, the initial symptoms can be listed as psychosis, dystonia-parkinsonism, and spasticity.

The brain MRIs of all patients were suggestive for iron accumulation equally at GP and SN. Five of them had hyperintense streaking of the medial medullary lamina, between the external and the internal part of the GP on T2 and FLAIR sequences. This radiological feature is not specific for a particular clinic. In patient M4, the medial streaking of GP was reminiscent of the 'eye of the tiger' sign common to PKAN (Fig. 2). Global brain atrophy was present in four patients, who also showed cognitive decline.

Optic atrophy, seizures and peripheral sensory-motor axonal neuropathy were the other clinical findings. Although optic atrophy was detected on examination in all patients, no patients had visual complaints. Patient M8 had a history of generalised seizures. Patient M6 had frontal intermittent rhythmic delta activity (FIRDA) in her EEG. Polyneuropathy was detected in two siblings from Family 3 with C19orf12 compound heterozygous variations. Generalised dystonia was the most prevalent symptom for patients M3 and M8. These patients died aged 29 and 34, respectively, due to status dystonicus.

\section{Discussion}

Herein, we present genetic analyses and associated clinical findings for seven C19orf12 variants identified in a cohort of 14 patients from 13 families with NBIA. Differential genetic diagnosis of MPAN was reached in seven patients from six families upon identification of biallelic C19orf12 variants. One patient had a monoallelic C19orf12 associated variant with inconsistent evaluations of pathogenicity (rs146170087). NBIA patients from the remaining six families with heterogeneous clinical findings were negative for potentially pathogenic C19orf12 variants, and were therefore noted as good candidates for exome sequencing for the identification of new genes for NBIA.

In our cohort with C19orf12 variants, the age of onset was dramatically clustered into two groups: MPAN started at ages 9 to 12 , or 22 to 27 . We wondered whether the variant 


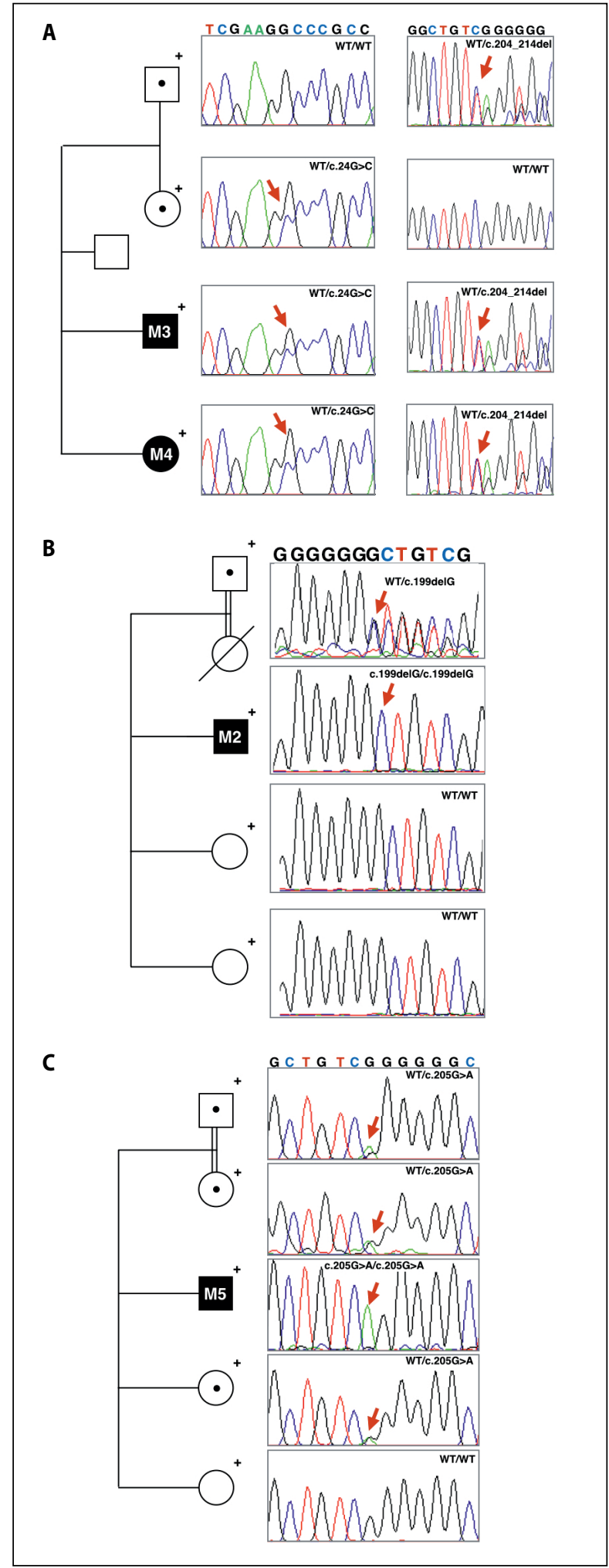

Figure 1. Segregation analysis in three families. (A) Patients M3 and M4 from Family 3 are found to be compound heterozygotes for c.24G > C (novel) and c.204_214del variants. (B) Patient M2 from Family 2 is homozygous for C.199delG variant, while his healthy sisters do not carry this variant. (C) Patient M5 from Family 4 is homozygous for c.205G > A. Parents and one of his sisters are carriers for the variant

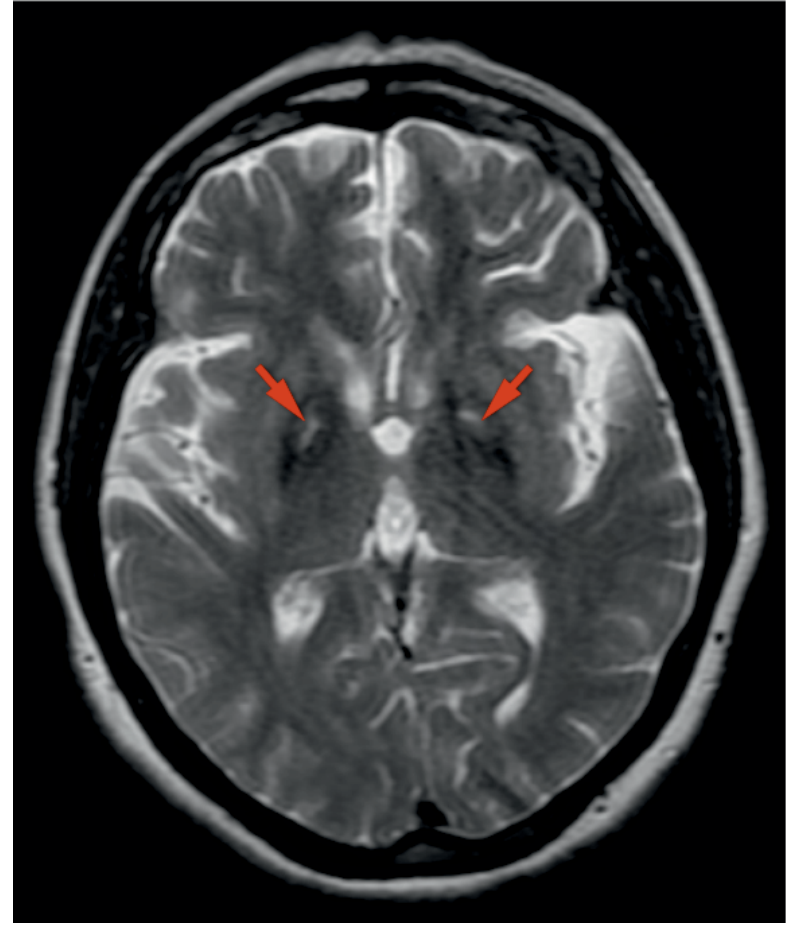

Figure 2. Brain MRI findings for Patient M4. Axial T2-weighted image of M4 shows hypointensities at bilateral globus pallidi indicative of iron accumulation. Red arrows mark abnormal hyperintense signals in the middle of the globus pallidus, which is known as medial streaking. Medial streaking is so marked in this patient that it could be confused with the 'eye of the tiger' sign

type had any impact on this strict assemblage. At first glance, both missense and frameshifting variants are found in the early-onset group. However, re-annotation of the variants with VEP revealed a potential splicing effect for variants c. $24 \mathrm{G}>\mathrm{C}$ and c.194G $>$ A, which alter the first nucleotide of exons 2 and 3, respectively. Interestingly, the missense variant c.205G > A (rs515726205) residing 12 bp into exon 3 has the potential to alter splicing, as suggested by the Human Splice Finder algorithm. A potential frameshifting effect by a splicing impact may be associated with earlier disease onset. RNA-based analyses are required in large cohorts with early-onset MPAN to test this effect on different C19orf12 transcripts. C19orf12 is a small gene with exons ranging from 2 to 4 in different. Therefore, it is not possible to predict if the frameshifting effect results in null alleles through the nonsense mediated decay (NMD) mechanism or truncated proteins.

The patients who carried variant $\mathrm{c} 24 \mathrm{G}>\mathrm{C}$, i.e. $\mathrm{M} 3$ and M4, showed a catastrophic disease progression characterised by cognitive decline, spasticity, and dystonia. Patient M3 died aged 36 due to a respiratory infection. His sister, M4, has been bedridden since her late twenties. In a patient with $\mathrm{c}$ 194G > A, M1, spasticity and dystonia were the prominent symptoms. Despite pharmacological treatment with baclofen, 
trihexyphenidyl and intra-muscular botulinum toxin injections, she underwent deep brain stimulation surgery at the age of 18. There is no MPAN-specific treatment option. The therapeutic effect of iron chelation with deferiprone in NBIA has not been proven. Treatment of spasticity, dystonia, and parkinsonism, as well as significant neuropsychiatric symptoms, are indicated in MPAN $[1,4,9]$.

In patients with NBIA, dystonia with spasticity, especially in the lower extremities, are frequent symptoms for childhood- and adolescence-onset MPAN. Dystonia and pyramidal symptoms are also prevalent motor symptoms common to both age groups. A late onset, but a rapidly progressive course with psychiatric symptoms and parkinsonism are features confined to adult-onset MPAN [4, $6,10]$. The rapid progressive course includes both motor deterioration and cognitive decline, which eventually leads patients to be bed-bound. The c.32C > T; p.(Thr11Met) variant has previously been reported as the most frequent variant in Turkish adult-onset MPAN cases [4]. In our cohort, we found this variant in two of our three adult-onset MPAN cases. At this point, we would like to emphasise the possibility of the misinterpretation of clinical findings of late-onset MPAN patients. For example, patient M6 with homozygous c.32C > T; p.(Thr11Met) variant was first sent to a psychiatry clinic due to catatonic psychosis. Further neurological evaluations revealed Parkinson-like findings with predominant dystonia. Only then was she assigned for MRI analysis which led to the identification of iron accumulation in the brain. She then received dopaminergic therapy with a good response. Adult-onset MPAN should also be distinguished from atypical neuroaxonal dystrophy (NAD) due to PLA2G6 mutations [5]. Both MPAN and atypical NAD show dystonia-parkinsonism and iron deposition at GP and SN. Atypical NAD differs from MPAN in terms of cerebellar findings including cerebellar atrophy and eye movement anomalies $[1,11]$. A correct genetic diagnosis will in turn assist in such differential diagnoses.

The third adult-onset MPAN case had an interesting genetic finding: we could only detect heterozygosity for a relatively common variant c.424A > G; p.(Lys142Glu) in C19orf12. This variant was originally reported to be pathogenic and found to be in a compound heterozygous state with c.205G > A; p.(Gly69Arg) [1]. However, since this original report, this variant has had conflicting interpretations of pathogenicity ranging from benign to pathogenic in ClinVar. Because this patient had characteristic adult-onset MPAN findings with fast progression and death only two years after the emergence of initial symptoms, we cannot exclude the possibility that an as yet unidentified variant, maybe a $\mathrm{CNV}$ event along with c.424A > G; p. (Lys142Glu), was responsible for his condition.

Pathogenic variations in C19orf12 are responsible for two allelic diseases with autosomal recessive inheritance: MPAN and spastic paraplegia (SPG) type 43 [12]. SPGs or hereditary spastic paraplegias (HSPs) are a heterogenous group of rare neurological disorders characterised by progressive lower extremity spasticity without apparent brain iron accumulation. The condition is designated as complicated HSP if spasticity is accompanied by additional features including cognitive impairment, cerebellar syndrome, thin corpus callosum, or neuropathy. In this sense, patient M5 with early-onset MPAN had a distinct and overlapping clinical course with complicated HSP. Our patient had slowly progressive spastic paraparesis without extrapyramidal signs. Distal muscle atrophy with weakness was present. He had knee flexion and Achilles tendon contractures. Nerve conduction study was normal at the age of 15 , but he had first motor neuron signs at needle electromyography. He had mild mental retardation and optic atrophy. There was no evidence for dystonia, parkinsonism or any other extrapyramidal findings. Therefore, his clinical picture was compatible with complicated HSP, except for the iron accumulation in his brain MRI (Fig. 3). Therefore, patient M5, who is currently 22 , has restricted the differential diagnosis of early-onset MPAN, as he has not yet developed either dystonia or parkinsonism.

Although MPAN demonstrates a wide phenotypical spectrum, the most common finding is iron accumulation equally in GP and SN and medial medullary lamina linear hyperintense streaking on T2 sequences. In some cases, such as patient M4 when the linear streaking is prominent, it resembles the 'eye of the tiger' sign (Fig. 2). This can lead to a misdiagnosis. In PKAN cases, the hyperintensity of GP is located in the anteromedial area of GP on a T2-weighted MRI. Global brain atrophy and spasticity may help to distinguish MPAN from PKAN. As shown in our cohort, most MPAN cases had brain atrophy and all had spasticity. Brain atrophy and spasticity are not features of the PKAN phenotype [9].

The C19orf12 variants caused a wide spectrum of overlapping phenotypes and histopathological findings. MPAN can present as juvenile ALS [3], HSP [12], Behr syndrome [13] and dystonia-parkinsonism [1]. Lewy bodies, tangles, spheroids, and tau pathology are known in MPAN histopathology. Cognitive decline, global brain atrophy, parkinsonism, and histopathological findings show another overlap between MPAN and other Lewy body and taupathy-associated neurodegenerative diseases [1]. The clinical involvement in MPAN is not uniform. Variable degrees of cognitive decline, pyramidal and extrapyramidal findings are seen in MPAN patients. The C19orf12 has been reported to code for a mitochondrial and endoplasmic reticulum membrane protein responsible for the transfer of essential lipids, and it has a role in calcium and magnesium metabolism and in autophagosome formation [12, 14]. New functional studies of C19orf12 will eventually shed light on the overlapping phenotypes such as HSP and Parkinson's and Alzheimer's diseases. 

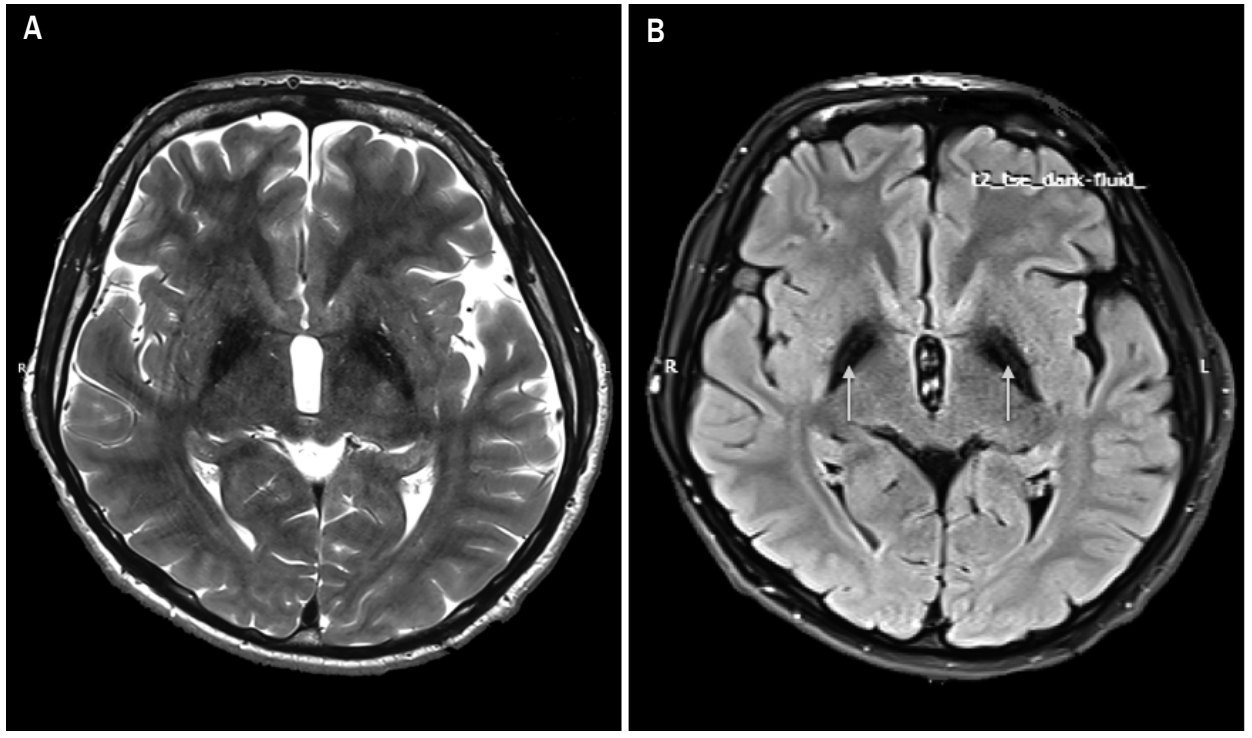

Figure 3. Brain MRI findings for Patient M5. (A) The T2 and (B) T2_tse_dark fluid weighted axial section images showing hypointensity at globus pallidi. White arrows indicate confluent medial streaking in the globus pallidus (B)

\section{Conclusion}

We present an MPAN series with a wide range of clinical and genetic findings. We have associated two pathogenic variants [c.24G > C; p.(Lys8Asn) and c.194G > A; p.(Gly65Glu)] with the MPAN phenotype for the first time. We have also supplied a genetic diagnosis of MPAN to a patient with atypical findings, who clinically resembled complicated HSP except for iron accumulation in his brain. The phenotypic presentation of this patient brought us to the conclusion that the clinical spectrum of MPAN may exclude dystonia and parkinsonism for at least some of the cases. The variant c.32C > T; p.(Thr11Met) common to Turkish adult-onset MPAN patients was also found to be predominant for genetically defined late-onset MPAN patients in our cohort. Finally, we would like to highlight the possibility that frameshifting variants could be associated with early-onset MPAN.

Ethics: This study was approved by the Ethics Committee of Istanbul Faculty of Medicine, Istanbul University (protocol number 2015/12837/1.015.223). Written informed consents were received for/from patients authorizing us to use their medical data in this publication.

Acknowledgments: The authors wish to thank the patients and their families for participating in this study. This work was supported by grant from the Scientific Research Projects Coordination Unit of Istanbul University, Project Numbers TSA-2018-27512 and TDK-2017-26646.

\section{References}

1. Hartig MB, luso A, Haack T, et al. Absence of an orphan mitochondrial protein, c19orf12, causes a distinct clinical subtype of neurodegene- ration with brain iron accumulation. Am J Hum Genet. 2011; 89(4): 543-550, doi: 10.1016/j.ajhg.2011.09.007, indexed in Pubmed: 21981780.

2. Schottmann G, Stenzel W, Lützkendorf S, et al. A novel frameshift mutation of C190RF12 causes NBIA4 with cerebellar atrophy and manifests with severe peripheral motor axonal neuropathy. Clin Genet. 2014; 85(3): 290-292, doi: 10.1111/cge.12137, indexed in Pubmed: 23521069

3. Deschauer M, Gaul C, Behrmann C, et al. C19orf12 mutations in neurodegeneration with brain iron accumulation mimicking juvenile amyotrophic lateral sclerosis. J Neurol. 2012; 259(11): 2434 2439, doi: 10.1007/s00415-012-6521-7, indexed in Pubmed: 22584950.

4. Olgiati S, Doğu O, Tufekcioglu Z, et al. The p.Thr11Met mutation in c19orf12 is frequent among adult Turkish patients with MPAN. Parkinsonism Relat Disord. 2017; 39: 64-70, doi: 10.1016/j.parkreldis.2017.03.012, indexed in Pubmed: 28347615.

5. Hogarth P, Gregory A, Kruer MC, et al. New NBIA subtype: genetic, clinical, pathologic, and radiographic features of MPAN. Neurology. 2013; 80(3): 268-275, doi: 10.1212/WNL.0b013e31827e07be, indexed in Pubmed: 23269600.

6. Dogu $\mathrm{O}$, Krebs $\mathrm{CE}$, Kaleagasi $\mathrm{H}$, et al. Rapid disease progression in adult-onset mitochondrial membrane protein-associated neurodegeneration. Clin Genet. 2013; 84(4): 350-355, doi: 10.1111/ cge.12079, indexed in Pubmed: 23278385.

7. Horvath R, Holinski-Feder E, Neeve VCM, et al. A new phenotype of brain iron accumulation with dystonia, optic atrophy, and peripheral neuropathy. Mov Disord. 2012; 27(6): 789-793, doi: 10.1002/ mds.24980, indexed in Pubmed: 22508347.

8. Skowronska M, Kmiec T, Kurkowska-Jastrzębska I, et al. Eye of the tiger sign in a 23 year patient with mitochondrial membrane protein associated neurodegeneration. J Neurol Sci. 2015; 352(12): 110-111, doi: 10.1016/j.jns.2015.03.019, indexed in Pubmed: 25819119

9. Akcakaya NH, Iseri SU, Bilir B, et al. Clinical and genetic features of PKAN patients in a tertiary centre in Turkey. Clin Neurol Neurosurg. 
2017; 154: 34-42, doi: 10.1016/j.clineuro.2017.01.011, indexed in Pubmed: 28113101.

10. Tschentscher A, Dekomien G, Ross S, et al. Analysis of the C19orf12 and WDR45 genes in patients with neurodegeneration with brain iron accumulation. J Neurol Sci. 2015; 349(1-2): 105-109, doi: 10.1016/j.jns.2014.12.036, indexed in Pubmed: 25592411.

11. Illingworth MA, Meyer E, Chong WK, et al. PLA2G6-associated neurodegeneration (PLAN): further expansion of the clinical, radiological and mutation spectrum associated with infantile and atypical childhood-onset disease. Mol Genet Metab. 2014; 112(2): 183189, doi: 10.1016/j.ymgme.2014.03.008, indexed in Pubmed: 24745848.
12. Landouré G, Zhu PP, Lourenço CM, et al. NIH Intramural Sequencing Center. Hereditary spastic paraplegia type 43 (SPG43) is caused by mutation in C19orf12. Hum Mutat. 2013; 34(10): 1357-1360, doi: 10.1002/humu.22378, indexed in Pubmed: 23857908.

13. Kleffner I, Wessling C, Gess B, et al. Behr syndrome with homozygous C190RF12 mutation. J Neurol Sci. 2015; 357(1-2): 115-118, doi: 10.1016/j.jns.2015.07.009, indexed in Pubmed: 26187298.

14. Venco P, Bonora M, Giorgi C, et al. Mutations of C19orf12, coding for a transmembrane glycine zipper containing mitochondrial protein, cause mis-localization of the protein, inability to respond to oxidative stress and increased mitochondrial $\mathrm{Ca}^{2}+$. Front Genet. 2015; 6: 185, doi: 10.3389/fgene.2015.00185, indexed in Pubmed: 26136767. 